\title{
Concurrencia de trastorno por consumo de sustancias y de otro trastorno mental en personas en tratamiento por alguno de ellos en España
}

\author{
Co-occurrence of substance use disorders and \\ other mental disorders in people undergoing \\ specific treatment for any of them in Spain
}

\author{
Juan José Fernández-Miranda*, Julio Fontoba-Díaz**, Silvia Díaz-Fernández*, \\ Francisco Pascual-Pastor***. \\ * AGC Salud Mental V. Hospital Universitario de Cabueñes, Gijón, Servicio de Salud del Principado de Asturias. \\ ** Hospital Regional Universitario Carlos de Haya, Málaga. \\ *** Unidad de conductas adictivas, Alcoi, Alicante.
}

\section{Resumen}

La concurrencia de trastornos por consumo de sustancias (TUS) y de otros trastornos mentales (OTM) es desconocida en su conjunto en España, y su abordaje se hace desde diferentes redes asistenciales. El objetivo del presente estudio era conocer la prevalencia de esta comorbilidad tanto en las redes de tratamiento de las adicciones como en las de salud mental. Se diseñó un estudio observacional, transversal, multicéntrico, con una muestra aleatorizada de pacientes en tratamiento por TUS u OTM en diferentes Comunidades Autónomas $(\mathrm{N}=1.783)$. Se completó un cuestionario ad hoc on-line recogiendo variables sociodemográficas, sobre uso de sustancias, y de diagnósticos de TUS y de OTM.

Los datos obtenidos en la encuesta arrojan una importante concurrencia de diagnósticos TUS y OTM. Se encontró una elevada prevalencia de OTM en aquellos en tratamiento en redes de adicciones por su TUS (71\%) y también tanto de diagnósticos de T.U. de cualquier sustancia $(68,9 \%)$ como de consumo activo de sustancias (50\%, exceptuando tabaco) en las personas en tratamiento por diagnósticos de OTM en redes de salud mental. Y, además, relaciones significativas entre la adicción a determinadas sustancias y trastornos mentales concretos: trastorno de personalidad con todos los TUS trastornos psicóticos con trastorno por uso de cannabis, pero no de cocaína; trastornos afectivos con trastornos por consumo de cocaína, y trastorno de ansiedad con trastorno por uso de cannabis. Este estudio aporta información preliminar sobre la elevada coexistencia en la práctica clínica habitual de trastornos adictivos y otros trastornos mentales en España, tanto en redes asistenciales de salud mental como específicas de adicciones.

Palabras clave: Prevalencia; comorbilidad; trastorno por consumo de sustancias; trastorno mental; consumo de sustancias.

\begin{abstract}
The co-occurrence of substance use disorders (SUD) and other mental disorders (OMD) is assumed to be high but is, in fact, unknown in Spain; and it is approached from different healthcare networks. The objective of the present study was to know the prevalence of this cooccurrence, both in specific addiction treatment networks and in mental health networks, in Spain. An observational, multicenter cross study, with a randomized sample, of patients under treatment for SUD or OMD in different Autonomous Communities of Spain was carried out $(\mathrm{N}=1,783)$. A specific ad hoc online questionnaire collecting sociodemographic variables, substance use and diagnoses of SUD and OMD was completed.

The data obtained in the survey show a significant concurrence of SUD and OMD diagnoses (in more than $60 \%$ of the patients). A high prevalence of OMD was found in those patients receiving treatment for their SUD (71\%), and also of diagnoses of any SUD $(68.9 \%)$ and active substance use $(50 \%$, except tobacco) in people receiving treatment for diagnoses of OMD. Also were found significant relationships between addiction to certain substances and specific mental disorders: personality disorders with all SUDs; psychotic disorders with cannabis use disorder, but not cocaine use disorder; affective disorders with cocaine use disorder, and anxiety disorders with cannabis use disorder. This study provides preliminary information about the high coexistence in routine clinical practice of addictive disorders and other mental disorders in different treatment settings in Spain.
\end{abstract}

Key words: Prevalence; comorbidity; substance use disorder; mental disorder; substance use.

Recibido: Mayo 2021; Aceptado: Septiembre 2021.

Enviar correspondencia a:

Juan José Fernández Miranda. Centro de tratamiento integral. AGC Salud Mental-SESPA. C/ Álava s/n, 33211, Gijón, Asturias.

E-mail: juanjofmiranda@gmail.com 
S e sabe que la comorbilidad psiquiátrica o concurrencia entre trastorno por uso/consumo de sustancias (TUS) y otros trastornos mentales (OTM) tiene una elevada prevalencia, aunque no conocida con precisión: Los estudios dan cifras dispares, según método diagnóstico, o si se trata de población general o en tratamiento (Hasin y Grant, 2015; Kessler y Merikangas, 2004; Regier et al., 1990). La importancia de esta comorbilidad radica no solo en esta alta prevalencia, sino también en su gravedad clínica y social, y su difícil manejo (Pascual-Pastor, Fernández-Miranda, Díaz-Fernández y Sala-Añó, 2017; Torrens, Mestre-Pintó y Domingo-Salvany, 2015).

En efecto, la concurrencia de TUS y OTM se supone alta pero no es conocida en España a nivel global, y no se dispone de ningún registro que permita delimitar cuantas personas cuentan con este doble diagnóstico ("diagnóstico dual”). Además, su abordaje se hace desde diferentes redes asistenciales (específica de adicciones y de salud mental), que presentan gran variabilidad. Y aunque es importante coordinar estrategias para la detección y el correcto tratamiento de pacientes con estos trastornos comórbidos, lo cierto es que un importante primer reto para el Sistema Nacional de Salud es conocer con la mayor concreción posible a qué se tiene que dar respuesta.

En España, aunque ya desde finales de los años noventa se han publicado estudios sobre la concurrencia de TUS y OTM, estos están realizados en general sobre poblaciones en tratamiento, con diagnósticos en casi todos ellos muy delimitados, y en concretas Comunidades Autónomas (CCAA), con diferentes modelos de atención (Arias, Ochoa y López-Ibor, 1996; Arias et al., 2013a; Fernández-Miranda, García-Portilla González, Sáiz Martínez, Gutiérrez-Cienfuegos y Bobes García, 2001; Lana, Fernández San Martín, Sánchez Gil y Bonet, 2008; Martínez-Gras et al., 2016; Pedrero-Pérez, 2018; Pereiro, Pino, Flórez, Arrojo y Becoña, 2013; Rodríguez-Llera et al., 2006; Roncero et al., 2011; Sánchez-Peña, Alvarez-Cotoli y Rodríguez-Solano, 2012), siendo quizás la excepción el estudio de Gual (2007). Es decir, hasta el momento no ha habido ningún estudio sobre prevalencia global de esta comorbilidad con claras características de nivel estatal y en ambas redes de tratamiento.

Para aproximarse al conocimiento de la prevalencia de la concurrencia de TUS con otros trastornos mentales en España a nivel asistencial, se diseñó un estudio relativo a personas en tratamiento en las redes de salud mental o de adicciones, en diferentes comunidades autónomas, y dentro de cada una de las redes en los diversos recursos en los que reciben tratamiento por sus TUS o por otros TM. Los objetivos del estudio eran averiguar el uso de sustancias entre los pacientes atendidos tanto en las redes específicas de adicciones como en las de salud mental, los diagnósticos de OTM añadidos al de TUS y los diagnósticos de TUS concomitantes al de OTM (comorbilidad entre trastornos). Y que relaciones existían entre los diagnósticos de trastornos por uso/consumo (TU) de diferentes sustancias y los OTM concretos.

\section{Método}

Estudio observacional, transversal, multicéntrico, con una muestra aleatorizada, de pacientes en tratamiento por trastornos adictivos $\mathrm{u}$ otros trastornos mentales en toda España $(\mathrm{N}=1.783)$. Un grupo de profesionales de salud completaron un cuestionario on-line, específicamente diseñado por los autores del estudio, sobre uso de sustancias y diagnósticos de sus pacientes en redes específicas de tratamiento de la adicción y de salud mental (unidades de desintoxicación, programas ambulatorios, hospitales/centros de día, unidades de agudos psiquiátricos, comunidades terapéuticas, unidades de media estancia y de rehabilitación, unidades/centros de salud mental, centros penitenciarios). La web de Socidrogalcohol alojó la encuesta ad hoc a la que podían acceder los profesionales para cumplimentar los datos de cada paciente (Variables recogidas: Tabla1), cumpliendo siempre con las máximas garantías de confidencialidad y anonimato. Los pacientes se anonimizaron desde el inicio.

La población de estudio eran personas en tratamiento en la red de salud mental o en la red de atención a adicciones en España, mayores de 18 años, y con un diagnóstico en el momento de la encuesta de trastorno por uso de sustancias o de otro trastorno mental. Se diseñó un muestreo no probabilístico, por conveniencia, y consecutivo. Se efectuó una aleatorización realizando la encuesta un mismo día a la semana, durante cuatro semanas consecutivas, sobre todos los pacientes que acudían a consulta del profesional ese día.

Las variables estudiadas fueron: Sociodemográficas (edad, sexo, situación laboral, convivencia), relativas a patologías somáticas (VHB, VHC, VIH), a uso de sustancias, y a diagnósticos tanto de trastorno por uso de sustancias como de otros trastornos mentales realizados por los profesionales que realizaban la encuesta (Tabla 1). La recogida de datos se realizó entre mayo de 2019 y mayo de 2020 en quince Comunidades Autónomas.

Se realizó una estadística descriptiva y otra inferencial. Para esta última se utilizó la chi cuadrado de Pearson (significación asintótica bilateral), con la prueba exacta de Fisher (significación exacta bilateral), para las variables cualitativas y las cuantitativas dicotomizadas. El intervalo de confianza se estableció en el 95\%. Para el procesamiento de los datos se utilizó el programa SPSS (versión v. 23).

El estudio se realizó conforme a los principios éticos de la Declaración de Helsinki.

\section{Resultados}

Se obtuvieron un total de 1.783 encuestas adecuadamente realizadas de las 2.000 previstas, llevadas a cabo por 
Tabla 1. Variables estudiadas.

\section{Recurso asistencial:}

Red adicciones: Centro específico de atención a adicciones/ Asociación de afectados/ Centros penitenciarios.

Red salud mental: Unidad Salud Mental Ambulatoria/ Unidad Hospitalaria / Asociación de afectados.

Sociodemográficas: Sexo, edad, estado civil, convivencia, situación laboral.

Otras patologías somáticas: Hepatitis C-Hepatitis B. VIH. Enf. Neurológica.

Sustancias consumidas en el último mes/Sustancias que ha consumido, pero no en el último mes: Tabaco/Alcohol/Cannabis/Cocaína/Estimulantes/

Heroína/Opiáceos de prescripción/Otras.

Trastornos mentales debidos al consumo de sustancias- últimos doce meses/ hace más de 12 meses:

F10. Trastorno por consumo de alcohol

F11. Trastorno por consumo de opioides

F12. Trastorno por consumo de cannabinoides

F13. Trastorno por consumo de sedantes o hipnóticos

F14. Trastorno por consumo de cocaína

F15. Trastorno por consumo de otros estimulantes

F16. Trastorno por consumo de alucinógenos

F17. Trastorno por consumo de tabaco

F18. Trastorno por consumo de disolventes volátiles

F19. Trastorno por consumo de múltiples drogas o de otras sustancias psicótropas

Otros trastornos mentales (agrupados):

F00-09 (Trastornos orgánicos)

F20-29 (Trastornos psicóticos)

F30-39 (Trastornos afectivos)

F40-49 (Trastornos de ansiedad)

F50-59 (Trastornos de la conducta alimentaria y del sueño)

F60-69 (Trastornos de personalidad)

F70-99 (Otros Trastornos)

61 profesionales en todas las CC.AA. excepto Cantabria y Navarra. De ellas, 322 en dispositivos de la red de salud mental (13 profesionales) y el resto en dispositivos de atención a trastornos adictivos (48 profesionales) (Tabla 2).

Hay más hombres y más jóvenes en la red de adicciones. Y están más activos laboralmente los pacientes en tratamiento en la red de adicciones, que además tienen tratamientos previos más frecuentemente, y donde las en-

Tabla 2. Número de encuestas por Comunidad Autónoma [Total (salud mental)].

\begin{tabular}{lcc}
\hline Comunidad Autónoma & Número (Salud mental) & Porcentaje \\
\hline Galicia & $281(44)$ & 15,8 \\
\hline C. Valenciana & $265(20)$ & 14,9 \\
\hline Baleares & $242(11)$ & 13,6 \\
\hline Asturias & $191(91)$ & 10,7 \\
\hline Madrid & $185(95)$ & 10,4 \\
\hline Castilla y León & $165(26)$ & 9,3 \\
\hline Andalucía & $110(8)$ & 6,2 \\
\hline Cataluña & $99(19)$ & 5,6 \\
\hline Aragón & 92 & 5,2 \\
\hline Canarias & 89 & 5,0 \\
\hline Castilla-La Mancha & $34(6)$ & 1,9 \\
\hline País Vasco & $11(2)$ &, 6 \\
\hline Extremadura & 7 &, 4 \\
\hline Murcia & 7 &, 4 \\
\hline Rioja & 5 & 100,0 \\
\hline TOTAL & 1.783 & \\
\hline
\end{tabular}

fermedades infecciosas (VIH, VHC) se detectan más. El consumo de alcohol se relaciona con el VHB y la presencia de enfermedad neurológica; el de opioides y de cannabis con la hepatitis C, B y el VIH; el de tabaco con la hepatitis C y las enfermedades neurológicas; el de cocaína con el VHC; y el de ansiolíticos con VHC y VIH.

Las sustancias más consumidas son, por este orden: Alcohol, tabaco, cannabis, cocaína y opioides. Hay mayor porcentaje de consumidores activos y pasados (abstinentes) en la red de adicciones, y un elevado porcentaje de pacientes con consumo de sustancias en la red de salud mental. Todas las sustancias son más consumidas en la red de adicciones salvo el alcohol, que lo es por igual en ambas redes. Aunque no está reflejado en la Tabla 4, existe un policonsumo en muchos casos, siendo el más habitual el de tabaco y alcohol (19,3\% de los sujetos) y de tabaco y cannabis (en el 6,8\%).

En la red de salud mental, un $68,9 \%$ de pacientes tiene un diagnóstico actual de TUS, más bajos que en la de adicciones. Destacan el trastorno por uso/consumo (TU) de alcohol, de tabaco, de cocaína y de cannabis en el total. En la red de adicciones son los TU de alcohol, tabaco y cocaína los más encontrados, mientras que en la red de salud mental lo son los TU de tabaco, alcohol y cannabis. Por otra parte, y no explicitado en la Tabla 5, se da una importante concurrencia de varios diagnósticos actuales por uso de sustancias, siendo los más frecuentes los de TU de tabaco y de alcohol $(10,6 \%)$ y de TU de cocaína y de alcohol (5,3\%).

Hay una muy elevada prevalencia de diagnósticos comórbidos en la red de adicciones, destacando en ella los trastornos afectivos y los de personalidad; en la red de 
Concurrencia de trastorno por consumo de sustancias y de otro trastorno mental en personas en tratamiento por alguno de ellos en España

Tabla 3. Características sociodemográficas y clínicas (somáticas) de la muestra.

\begin{tabular}{|c|c|c|c|c|}
\hline & $\begin{array}{c}\text { TOTAL } \\
N=1783\end{array}$ & $\begin{array}{l}\text { S. MENTAL } \\
N=322\end{array}$ & $\begin{array}{l}\text { ADICCIONES } \\
\qquad N=1461\end{array}$ & Valores de $F ; \chi^{2}, p$ \\
\hline Sexo (Hombres) & $1310(73,6 \%)$ & $216(67,3 \%)$ & 1094 (75\%) & 7,$67 ; 0,005$ \\
\hline Edad* & $47,54 \pm 1,38$ & $48,30 \pm 12,17$ & $43,11 \pm 11,21$ & 1,$79 ; 0,04$ \\
\hline Convivencia (Fam.prop+solo) & $1049(33+25,9 \%)$ & $34,5+27,3 \%$ & $25,5+32,6 \%$ & 1,$08 ; 0,75$ \\
\hline Situación laboral (Activo+IT) & $591(29,3 \%)$ & $89(20,8 \%)$ & $462(31,2 \%)$ & 5,$38 ;<, 0001$ \\
\hline Estado civil (Soltero) & $908(50,9 \%)$ & $168(52,2 \%)$ & $740(50,7 \%)$ & 1,$51 ; 0,624$ \\
\hline $\mathrm{VHC}$ & $292(16,4 \%)$ & $27(8,4 \%)$ & $265(18,1 \%)$ & 4,$97 ;<, 0001$ \\
\hline VHB & $65(3,6 \%)$ & $7(2,2 \%)$ & $58(4 \%)$ & 1,$05 ; 0,120$ \\
\hline $\mathrm{VIH}$ & $89(5 \%)$ & $5(1,6 \%)$ & $84(5,7 \%)$ & 8,$12 ; 0,002$ \\
\hline Enfermedad neurológica & $71(4 \%)$ & $15(4,7 \%)$ & $56(3,8 \%)$ & 0,$07 ; 0,493$ \\
\hline
\end{tabular}

*: Media, desviación estándar; Fam prop: Familia propia; IT: Incapacidad laboral.

Tabla 4. Prevalencia de las sustancias consumidas (Total, red de salud mental y red de adicciones [N (\%)].

\begin{tabular}{|c|c|c|c|c|}
\hline & $\begin{array}{c}\text { TOTAL } \\
N=1783\end{array}$ & $\begin{array}{l}\text { S. MENTAL } \\
N=322\end{array}$ & $\begin{array}{c}\text { ADICCIONES } \\
N=1461\end{array}$ & Valores de $\chi^{2} ; p$ \\
\hline Cualquiera previamente & $1287(72,2 \%)$ & $251(78 \%)$ & $1036(70,9 \%)$ * & 3,$85 ; 0,011$ \\
\hline Cualquiera menos tabaco previo & $1258(70,6 \%)$ & $237(73,6 \%)$ & $1021(69,9 \%)$ & 1,$06 ; 0,185$ \\
\hline Cualquiera actual & $1482(83,1 \%)$ & $250(77,6 \%)$ & $1232(84,3 \%)$ & 7,$67 ; 0,004$ \\
\hline Cualquiera menos tabaco actual & $1044(58,6 \%)$ & $161(50 \%)$ & $883(60,4 \%)$ & 9,$03 ; 0,001$ \\
\hline \multicolumn{5}{|l|}{ Sustancias consumidas último mes } \\
\hline Tabaco & $1195(89,1 \%)$ & $215(80,2 \%)$ & $980(91,3 \%)$ & 10,$03 ;<, 0001$ \\
\hline Alcohol & $693(50,3 \%)$ & $129(48,7 \%)$ & $564(50,6 \%)$ & 0,$08 ; 0,568$ \\
\hline Cannabis & $413(45,3 \%)$ & $49(31,6 \%)$ & $364(48,1 \%)$ & 16,$78 ;<, 0001$ \\
\hline Cocaína & $331(33,6 \%)$ & $27(20 \%)$ & $304(35,8 \%)$ & 12,$11 ;<, 0001$ \\
\hline Estimulantes (Anfetaminas) & $31(1,7 \%)$ & $12(3,7 \%)$ & $19(1,3 \%)$ & 7,$97 ; 0,003$ \\
\hline Heroína & $117(8,5 \%)$ & $4(1,5 \%)$ & $113(10,1 \%)$ & 25,$64 ;<, 0001$ \\
\hline Opioides de prescripción & $191(9,3 \%)$ & $5(1,4 \%)$ & $186(18,3 \%)$ & 10,$06 ; 0,002$ \\
\hline \multicolumn{5}{|c|}{ Sustancias consumidas, pero no en el último mes (abandono consumo) } \\
\hline Tabaco & $146(10,9 \%)$ & $53(19,8 \%)$ & $9(8,7 \%)$ & 11,$93 ;<, 0001$ \\
\hline Alcohol & $686(49,7 \%)$ & $136(51,3 \%)$ & $550(49,4 \%)$ & 1,$16 ; 0,158$ \\
\hline Cannabis & $498(54,7 \%)$ & $106(68,4 \%)$ & $392(51,9 \%)$ & 13,$76 ;<, 0001$ \\
\hline Cocaína & $654(66,4 \%)$ & $108(80 \%)$ & $546(64,2 \%)$ & 9,$89 ;<, 0001$ \\
\hline Estimulantes (Anfetaminas) & $260(14,6 \%)$ & $73(22,7 \%)$ & $187(12,8 \%)$ & 19,$31 ;<, 0001$ \\
\hline Heroína & $253(18,3 \%)$ & $24(9,1 \%)$ & $229(20,6 \%)$ & 20,$08 ;<, 0001$ \\
\hline Opioides de prescripción & $44(18,7 \%)$ & $6(54,5 \%)$ & 38 (17\%) & 22,$13 ;<, 0001$ \\
\hline
\end{tabular}

*: Resto no especificado o no consta.

salud mental son los trastornos psicóticos, además de los afectivos, los más habitualmente diagnosticados. Es en los trastornos psicóticos donde hay una diferencia significativa en su diagnóstico entre ambas redes.

Las relaciones halladas entre los TU de sustancias concretas y los diferentes TM específicos más relevantes son las siguientes: TU alcohol con T. afectivos; TU opioides con T. de personalidad (TP); TU cannabinoides con T. psicóticos, TP y de T. de ansiedad; TU sedantes con TP, T. de ansiedad y T. de sueño; TU cocaína con T. afectivos y TP, no relacionándose con trastornos psicóticos; TU estimulantes (anfetaminas) con T. psicóticos y con TP; y TU tabaco con T. psicóticos y TP.

En lo que respecta a los trastornos mentales no TUS, las relaciones más significativas son: Trastornos psicóticos con TU cannabis, otros estimulantes y tabaco, y en menor medida con alcohol; T. afectivos especialmente con TU cocaína y menos con TU Alcohol; T. ansiedad con TU cannabis y menos significativo con TU sedantes. Los trastornos de 
Juan José Fernández-Miranda, Julio Fontoba-Díaz, Silvia Díaz-Fernández, Francisco Pascual-Pastor

Tabla 5. Diagnóstico de trastorno por uso/consumo de sustancias (Total, red de salud mental y red de adicciones [N (\%)].

\begin{tabular}{|c|c|c|c|c|}
\hline & $\begin{array}{c}\text { TOTAL } \\
N=1783\end{array}$ & $\begin{array}{l}\text { S. MENTAL } \\
N=322\end{array}$ & $\begin{array}{c}\text { ADICCIONES } \\
N=1461\end{array}$ & Valores $\chi^{2} ; p$ \\
\hline Cualquiera previo & $1431(80,3 \%)$ & $215(66,8 \%)$ & $1216(83,2 \%)$ * & 16,$07 ;<, 0001$ \\
\hline Cualquiera actual & $1036(58,1 \%)$ & $222(68,9 \%)$ & $814(55,7 \%)$ & 14,$21 ;<, 0001$ \\
\hline Cualquiera previo (Menos T.U. tabaco) & $875(49,1 \%)$ & $190(59 \%)$ & $685(46,9 \%)$ & 11,$96 ;<, 0001$ \\
\hline Cualquiera actual (Menos T.U. tabaco) & $643(36,1 \%)$ & $142(44,1 \%)$ & $501(34,3 \%)$ & 8,$68 ; 0,002$ \\
\hline \multicolumn{5}{|c|}{ Trastorno por uso/consumo de sustancias en los últimos 12 meses } \\
\hline Alcohol & $744(41,7 \%)$ & $80(24,8 \%)$ & $664(45,4 \%)$ & 18,$32 ;<, 0001$ \\
\hline Opioides & $370(20,8 \%)$ & $12(3,7 \%)$ & $358(24,5 \%)$ & 28,$93 ;<, 0001$ \\
\hline Cannabinoides & $416(23,3 \%)$ & $41(12,7 \%)$ & $375(25,7 \%)$ & 13,$11 ;<, 0001$ \\
\hline Sedantes/Hipnóticos & $116(6,5 \%)$ & $10(3,1 \%)$ & $106(7,3 \%)$ & 15,$07 ;<, 0001$ \\
\hline Cocaína & $521(29,2 \%)$ & $33(10,2 \%)$ & $488(33,4 \%)$ & 18,$81 ;<, 0001$ \\
\hline Estimulantes (Anfet.) & $45(2,5 \%)$ & $12(3,7 \%)$ & $33(2,3 \%)$ & 1,$02 ; 0,128$ \\
\hline Alucinógenos & $5(0,3 \%)$ & 0 & $5(0,3 \%)$ & 0,$96 ; 0,293$ \\
\hline Tabaco & $643(36,1 \%)$ & $142(44,1 \%)$ & $501(34,3 \%)$ & 8,$98 ; 0,001$ \\
\hline Múltiples drogas & $36(2 \%)$ & $6(1,9 \%)$ & $30(2,1 \%)$ & 1,$91 ; 0,826$ \\
\hline \multicolumn{5}{|c|}{ Trastorno por uso/consumo sustancias previo a últimos 12 meses (abstinentes en la actualidad) } \\
\hline Alcohol & $498(27,9 \%)$ & $93(28,9 \%)$ & $405(27,7 \%)$ & 1,$52 ; 0,674$ \\
\hline Opioides & $305(17,1 \%)$ & $22(6,8 \%)$ & $283(19,4 \%)$ & 18,$75 ;<, 0001$ \\
\hline Cannabinoides & $355(19,9 \%)$ & $71(22 \%)$ & $284(19,4 \%)$ & 0,$66 ; 0,288$ \\
\hline Sedantes/Hipnóticos & $109(6,1 \%)$ & $6(1,9 \%)$ & $103(7 \%)$ & 22,$91 ;<, 0001$ \\
\hline Cocaína & $505(28,3 \%)$ & $66(20,5 \%)$ & $439(30 \%)$ & 6,$99 ; 0,001$ \\
\hline Estimulantes (Anfet.) & $81(4,5 \%)$ & $26(8,1 \%)$ & $55(3,8 \%)$ & 10,$87 ; 0,001$ \\
\hline Alucinógenos & $23(1,3 \%)$ & $1(0,3 \%)$ & $22(1,5 \%)$ & 3,$48 ; 0,085$ \\
\hline Tabaco & $347(19,5 \%)$ & $98(30,4 \%)$ & $249(17 \%)$ & 10,$16 ;<, 0001$ \\
\hline Múltiples drogas & $45(2,5 \%)$ & $16(5 \%)$ & $29(2 \%)$ & 8,$49 ; 0,002$ \\
\hline
\end{tabular}

*: Resto no especificado o no consta.

Tabla 6. Diagnóstico de otro trastorno mental (no F 10-19) (Total, red de salud mental y red de adicciones [N (\%)].

\begin{tabular}{|c|c|c|c|c|}
\hline & $\begin{array}{c}\text { TOTAL } \\
N=1783\end{array}$ & $\begin{array}{l}\text { S. MENTAL } \\
N=322\end{array}$ & $\begin{array}{c}\text { ADICCIONES } \\
N=1461\end{array}$ & Valores $\chi^{2} ; p$ \\
\hline CUALQUIERA & $1334(74,8 \%)$ & $297(92,2 \%)$ * & $1037(71,0 \%)$ & 10,$06 ;<, 0001$ \\
\hline PSICÓTICOS (F 20-29) & $315(17,7 \%)$ & $113(35,1 \%)$ & $202(13,8 \%)$ & 14,$02 ;<, 0001$ \\
\hline AFECTIVOS (F 30-39) & $580(32,5 \%)$ & $108(33,5 \%)$ & $472(32,3 \%)$ & 0,$06 ; 0,669$ \\
\hline ANSIEDAD (F 40-49) & $296(16,6 \%)$ & $43(13,4 \%)$ & $253(17,3 \%)$ & 1,$21 ; 0,084$ \\
\hline TCA y SUEÑO (F 50-59) & $70(3,9 \%)$ & $10(3,1 \%)$ & $60(4,1 \%)$ & 0,$09 ; 0,402$ \\
\hline PERSONALIDAD(F60-69) & $459(25,7 \%)$ & $71(22 \%)$ & $388(26,6 \%)$ & 1,$16 ; 0,094$ \\
\hline OTROS (F 00-09, 70-99) & $75(4,2 \%)$ & $7(2,1 \%)$ & $68(4,7 \%)$ & 3,$08 ; 0,042$ \\
\hline
\end{tabular}

*: Resto no especificado o no consta.

personalidad se relacionan con todos los TUS, pero especialmente con TU opioides, TU cannabis, TU sedantes, TU cocaína y TU tabaco.

Los resultados se presentan resumidos en las tablas 3 a 7 (En negrita los valores de $\mathrm{p}<, 05$ ). Las características sociodemográficas y clínicas somáticas se resumen en la Tabla 3. Las sustancias consumidas tanto en pacientes en la red de salud mental como en la de adicciones en la Tabla 4. Y los TUS, en la Tabla 5. Los diagnósticos de trastornos mentales no debidos al consumo de sustancias quedan resumidos en la Tabla 6. Por último, las relaciones encontradas entre los trastornos por consumo de diferentes sustancias y los otros diagnósticos de trastornos mentales, agrupados por grandes síndromes, quedan expuestos en la Tabla 7 (No se 
Tabla 7. Trastorno por uso de sustancias y otro trastorno mental (Valores de p).

\begin{tabular}{lcccccccc}
\hline $\mathbf{N}=1783$ & $\begin{array}{c}\mathrm{F}-10 \\
\text { ALCOHOL }\end{array}$ & $\begin{array}{c}\mathrm{F}-11 \\
\text { OPIOIDES }\end{array}$ & $\begin{array}{c}\mathrm{F}-12 \\
\text { CANNABIS }\end{array}$ & $\begin{array}{c}\mathrm{F}-13 \\
\text { SEDANTES }\end{array}$ & $\begin{array}{c}\mathrm{F}-14 \\
\text { COCAÍNA }\end{array}$ & $\begin{array}{c}\mathrm{F}-15 \\
\text { OTR ESTIM. }\end{array}$ & $\begin{array}{c}\mathrm{F}-16, \mathbf{1 8 , 1 9} \\
\text { OTR DRGS. }\end{array}$ & $\begin{array}{c}\mathrm{F} 17 \\
\text { TABACO }\end{array}$ \\
\hline F0-9. T. ORGÁNICOS & 0,468 & 0,716 & 0,435 & 0,803 & 0,090 & $\mathbf{0 , 0 2 0}$ & 0,821 & 0,216 \\
F20-29. T PSICÓTICOS & $\mathbf{0 , 0 1 2}$ & 0,188 & $\mathbf{0 , 0 0 0}$ & 0,594 & 0,739 & $\mathbf{0 , 0 0 0}$ & 0,866 & $\mathbf{0 , 0 0 0}$ \\
F30-39. T AFECTIVOS & $\mathbf{0 , 0 0 1}$ & 0,112 & 0,276 & 0,079 & $\mathbf{0 , 0 0 0}$ & 0,079 & 0,562 & 0,161 \\
F 40-49. T ANSIEDAD & 0,633 & 0,093 & $\mathbf{0 , 0 0 6}$ & $\mathbf{0 , 0 1 3}$ & 0,693 & 0,736 & 0,880 & 0,424 \\
F 50-59. TCA Y T SUEÑO & 0,638 & 0,609 & 0,170 & 0,541 & $\mathbf{0 , 0 4 6}$ & 0,164 & 0,100 & $\mathbf{0 , 0 4 4}$ \\
F 60-69. T PERSONALIDAD & $\mathbf{0 , 0 3 3}$ & $\mathbf{0 , 0 0 0}$ & $\mathbf{0 , 0 0 0}$ & $\mathbf{0 , 0 0 0}$ & $\mathbf{0 , 0 0 0}$ & $\mathbf{0 , 0 3 1}$ & 0,757 & $\mathbf{0 , 0 0 0}$ \\
F 70-99. OTROS T M & 0,597 & 0,079 & $\mathbf{0 , 0 3 6}$ & 0,690 & $\mathbf{0 , 0 1 9}$ & 0,129 & 0,831 & $\mathbf{0 , 0 0 9}$ \\
\hline
\end{tabular}

(G.L.=1; valores de $\chi^{2}$ Pearson entre 0,03 y 20,66).

exponen en función de red asistencial al no hallarse diferencias significativas por ello).

\section{Discusión}

\section{Conocimiento del problema en España}

Se estima que más de un tercio de las personas diagnosticadas de un trastorno mental abusa o es dependiente de sustancias psicoactivas, especialmente el alcohol, y entre los pacientes dependientes de sustancias al menos un porcentaje semejante presenta otros trastornos mentales (Conway, Compton, Stinson y Grant, 2006; Grant et al., 2004; Hasin y Grant, 2015; Hunt, Large, Cleary, Lai y Saunders, 2018; Kessler y Merikangas, 2004; Lev-Ran, Imtiaz, Rehm y Le Foll, 2013; Regier et al., 1990). La prevalencia de la comorbilidad difiere entre la población general y la que está en tratamiento, siendo más elevada en esta última (Pascual-Pastor et al., 2017; Torrens et al., 2015). Numerosos estudios señalan las altas tasas de diagnóstico dual en los pacientes en tratamiento en las redes de salud mental y de adicciones (Compton, Thomas, Stinson y Grant, 2007; Hunt et al., 2018; Lev-Ran et al., 2013; Pascual-Pastor et al., 2017).

En España, ya Arias et al. (1996) estudian los trastornos de personalidad (TP) en dependientes de opiáceos en tratamiento con naltrexona; Fernández-Miranda et al. (2001) exploran la influencia de los trastornos psiquiátricos en la efectividad de un programa de mantenimiento con metadona; Haro, Bolinches y De Vicente (2001) estudian la influencia de los TP en la evolución de la conducta adictiva y psicopatología asociada, al igual que Fernández-Miranda y Gutiérrez-Cienfuegos (2005). Rodríguez-Llera et al. (2006) averiguan la prevalencia de concurrencia de trastornos mentales en consumidores de heroína. Otras investigaciones se focalizan en trastornos mentales específicos, especialmente los de personalidad y su concomitancia con trastornos adictivos (Fernández-Miranda, 2002; Lana et al., 2008; Pedrero-Pérez, 2018). Herrero, Domingo-Salvany, Torrens y Brugal (2008) determinan la comorbilidad psiquiátrica en consumidores de cocaína, al igual que Martí-
nez-Gras et al. (2016). Roncero et al. (2011) estudian las comorbilidades psiquiátricas en pacientes en tratamiento con metadona en diversas CCAA. En el estudio de Gual (2007) sobre 2.361 pacientes con TUS en tratamiento en redes de adicciones en varias CC.AA., el 33,8\% presentaban un diagnóstico dual. La depresión era el trastorno más prevalente $(21,6 \%)$, seguido de los trastornos de ansiedad $(11,7 \%)$. Los dependientes de alcohol que además abusaban de otras drogas mostraron más comorbilidad (48,5\%), y los adictos a los opiáceos los que menos $(27,4 \%)$.

En el estudio de Arias et al. (2013a), el 61,8\% de los pacientes tenían diagnóstico actual de un trastorno mental distinto al TUS. En el de Pereiro et al. (2013), se halló que el 56,3\% de pacientes con TUS también tenían además un diagnóstico de otro trastorno mental. Ya específicamente, Cuenca et al. (2013) y Comín et al. (2016) estudian la comorbilidad psiquiátrica en consumidores de cannabis y en dependientes de cocaína; y Araos et al. (2017) en pacientes con trastorno por consumo de cocaína. Todos encuentran importantes prevalencias.

Por otra parte, en un estudio en unidad hospitalaria (agudos) de psiquiatría (Rodríguez-Jiménez et al., 2008), el 24,9\% de los pacientes ingresados presentó un TUS (excluyendo dependencia a nicotina) concurrente. En una muestra de pacientes de centros de salud mental, más del $50 \%$ de los atendidos presentaban además un trastorno adictivo (Sánchez-Peña et al., 2012).

Estos estudios aportan datos sobre la situación de la coexistencia de TUS y OTM parciales, pero que apuntan a algunos de los hallazgos de nuestra investigación, que no confirman otros.

\section{Perfil sociodemográfico y clínico, y de uso de sustancias}

Las características sociodemográficas de nuestra muestra, en lo que se refiere al perfil de las personas tratadas en la red de adicciones, no difieren demasiado del ya conocido (Arias et al., 2013a; Gual, 2007; Pascual-Pastor et al., 2017; Torrens et al., 2015), lo que avala la representativi- 
dad de la muestra en este sentido. En cuanto a la situación clínica somática, es lógico haber encontrado mayor prevalencia de infecciones por VHC y VIH en la red de adicciones, y que los tratamientos previos por TUS sean más frecuentes en los centros de tratamiento de adicciones. Las hepatitis B y C, y el VIH, son más frecuentes en hombres que en mujeres, y podría reflejar más conductas de riesgo por parte de los varones. Para el caso de la hepatitis $\mathrm{C}$ y el VIH, existe una relación con la heroína, al igual que con los opioides de prescripción, y también con el cannabis y los ansiolíticos. Esto podría ser explicado por el policonsumo de diversas drogas (Arias et al., 2013a; Fernández-Miranda et al., 2001).

Las sustancias más consumidas en ambas redes son, por este orden, alcohol, tabaco, cocaína, cannabis y opioides, lo que se corresponde en gran medida con lo conocido sobre poblaciones en tratamiento. No sorprende que haya mayor porcentaje tanto de consumidores activos como de ya abstinentes en la red de adicciones, pero sí el elevado porcentaje de consumo de sustancias en la red de salud mental (casi la mitad, si no se incluye tabaco). También destaca que, aunque obviamente todas las sustancias son más consumidas en la red de adicciones, la excepción sea el alcohol, que lo es por igual en ambas redes. No obstante, esto podría reflejar distorsiones en la atención a los problemas con el alcohol, en comparación con el resto de sustancias de abuso, en la red de adicciones (Arias et al., 2013b; Flórez-Menéndez, Balcells-Oliveró y Uzal-Fernández, 2018; Smith y Randall, 2012).

Menos para el alcohol, se produjo un mayor abandono de uso de sustancias por parte de los pacientes en la red de salud mental que en la de adicciones, lo que podría ser consecuencia de una menor gravedad en su uso, o de la mejoría del OTM (“automedicación”) (Arias et al., 2013a; Torrens et al., 2015).

\section{Diagnósticos de TUS y OTM. Comorbilidad}

En nuestro estudio es llamativo que en la red de salud mental un $66,9 \%$ de pacientes tiene un diagnóstico actual de TUS (44,1\% si no se considera tabaco), porcentaje que es más bajo en la red de adicciones (explicable al estar recibiendo tratamiento específico para la abstinencia en ella). Destacan como trastornos más prevalentes el TU tabaco $(89,1 \%)$, TU alcohol $(24,8 \%)$, TU cannabis $(12,7 \%)$ y TU cocaína $(10,2 \%)$, lo que no difiere mucho otras investigaciones previas (Arias et al., 2016; Fernández-Miranda et al., 2001; Ochoa-Mangado, Molins-Pascual y Seijo-Ceballos, 2018; Welsh et al., 2017). Hay más diagnósticos relacionados con el TU tabaco en pacientes tratados en la red de salud mental que en la de adicciones $(44,1 \%$ vs. $34,3 \%)$, aunque podría no reflejar exactamente la realidad y estar condicionado por una mayor preocupación por ese consumo en la red de salud mental que en la de adicciones (Becoña et al., 2006).
En lo relativo a los diagnósticos por otros trastornos mentales, el hallazgo fundamental es que hay un $71 \%$ de pacientes diagnosticados con otro trastorno mental diferente al adictivo en la red de adicciones. También mostraría la sensibilidad de los profesionales con el problema de la concurrencia de diagnósticos, y su amplia detección. El que sean mayoritarios los trastornos afectivos $(32,3 \%)$, los de personalidad $(26,6 \%)$, los de ansiedad (17,3\%) y los psicóticos $(13,8 \%)$ concuerda con lo mostrado en general por algunos estudios previos (Arias, et al., 2013a; Farren, Hill y Weiss, 2012; Fernández-Miranda et al., 2001; Grant et al., 2004; Lev-Ran et al., 2013; Nocon, Bergé, Astals, Martín-Santos y Torrens, 2007). Cabe destacar que, menos los trastornos psicóticos, que son algo menores, los porcentajes de los diferentes trastornos mentales tratados son semejantes a los de la red de salud mental, afianzando con esto la percepción de la capacidad de la red de adicciones de detectar otros trastornos mentales (Arias et al., 2013a; Ochoa-Mangado et al., 2018; Pereiro et al., 2013).

En lo que respecta a las relaciones encontradas entre concretos TUS y específicos OTM, destaca que los trastornos psicóticos se relacionan con TU cannabis, pero no con TU cocaína, aunque sí con otros estimulantes, lo que no está en línea con lo habitualmente hallado (Arias et al., 2013c; Herrero et al., 2008; Hunt et al., 2018; Martínez-Gras et al., 2016). Es más habitual la relación con TU tabaco en primer lugar, y en menor medida con alcohol (Arias et al., 2013b; Flórez-Menéndez et al., 2018; Pascual-Pastor et al., 2017). Los trastornos afectivos se relacionan especialmente con el TU cocaína, lo que es habitual, especialmente en los trastornos bipolares (Araos et al., 2017; Arias et al., 2016; Herrero et al., 2008); pero ya no tanto que lo hagan menos con el TU alcohol (Araos et al., 2017; Arias et al., 2013b; Flórez-Menéndez et al., 2018). En cuanto a los trastornos de ansiedad, es un hallazgo menos claro de explicar su relación con el TU cannabis (Conway et al., 2006; Fatséas, Denis, Lavie y Auriacombe, 2010; Grant et al., 2004; Sáiz Martínez et al., 2014; Smith y Randall, 2012); también es llamativo que la relación sea menos significativa con el TU sedantes, aunque este trastorno esté condicionado y solapado con la propia prescripción de estos fármacos, sin poder aclarar hasta que puto exista un abuso o dependencia de los mismos (Fernández-Miranda, Orengo-Caus y Díaz-Fernández, 2019; Guardia-Serecigni y Flórez-Menéndez, 2018; Pereiro et al., 2013; Sáiz Martínez et al., 2014).

Es muy clarificador como los trastornos de personalidad se relacionan significativamente con todos los TUS, pero especialmente con el TU opioides (Fernández-Miranda y Gutiérrez-Cienfuegos, 2005; Fernández-Miranda et al., 2019), TU cannabis, TU sedantes, TU cocaína (Haro et al., 2001; Pedrero-Pérez, 2018) y TU tabaco (Becoña et al., 2006); y en menor medida con TU alcohol y TU otros estimulantes (Arias et al., 2013b; 2013c). La relación entre el hecho de tener un TP y el consumo problemático de 
drogas se confirma claramente en nuestro estudio. Y también que ese consumo lo es de muy diferentes sustancias, sin poder concretar una específica claramente relacionada con este trastorno (Arias et al., 2013a; Fernández-Miranda, 2002; Pedrero-Pérez, 2018).

\section{Fortalezas y limitaciones del estudio}

Esta investigación es la primera que se realiza en nuestro país con un claro objetivo de ámbito nacional, incluyendo a casi todas las CC.AA. Y también reflejando la diversidad de redes asistenciales. La muestra tiene un número importante de sujetos, y además se ha sometido a una aleatorización. Se ha utilizado un enfoque descriptivo y una definición inclusiva de comorbilidad. Con todo ello, puede considerarse como representativa en buena medida de las personas con diagnósticos de TUS y otros TM concurrentes, en la práctica asistencial habitual, en toda España. En definitiva, las principales fortalezas de este estudio son el tamaño de la muestra, el alcance nacional y la variedad de entornos de tratamiento, para evitar en lo posible sesgos; en última instancia, su aceptable representatividad.

Una posible limitación de este estudio es la inclusión de casos, que no se realizó de manera uniforme y podría haber tenido sesgos en la recolección de la muestra, especialmente el hecho de que haya menos representación de pacientes tratados en la red de salud mental que de la de adicciones. También podría tener un sesgo porque las propias estructuras asistenciales en las distintas CC.AA. son muy heterogéneas. Pero, por otra parte, esta es la realidad de la atención a los TUS (y en parte también a los trastornos mentales) en el estado español. Otra posible limitación podría ser también el método de aleatorización, del que ya se ha comentado sus particularidades, pero que ha sido elegido por su facilidad de aplicación. Por otro lado, no se han utilizado cuestionarios validados, sino uno construido ad hoc, de fácil cumplimentación y recogiendo las variables más relevantes para el objetivo del estudio.

Por último, hay que señalar que la comparabilidad con otros estudios es limitada, ya que, como se ha comentado, apenas existen estudios en nuestro medio de carácter tan general.

\section{Conclusiones}

Esta investigación puede proporcionar, dadas las características de su diseño (intentando evitar varios sesgos), aunque también considerando sus limitaciones, una información preliminar, aunque orientativa y valiosa, sobre la prevalencia de concurrencia de diagnósticos de TUS y de OTM tanto en la red de salud mental como, especialmente, en la de adicciones, en todo el país. Aunque hay que tener presente que ha habido una menor respuesta en la red de salud mental a las encuestas (Que podría reflejar una menor conciencia del problema en esa red que, his- tóricamente y hasta tiempos recientes, se ha caracterizado por una baja exploración de los trastornos adictivos que podían tener los pacientes atendidos por otro trastorno mental en esos servicios).

En general, los datos obtenidos arrojan una importante concurrencia de diagnósticos (en más del $60 \%$ de los pacientes). Muestran la existencia de una elevada prevalencia de otros trastornos mentales en aquellos pacientes a tratamiento por su trastorno adictivo y, quizá de forma más sorprendente, una muy alta prevalencia tanto de diagnósticos de TUS como de consumo activo de sustancias en las personas en tratamiento por diagnósticos de otros trastornos mentales en los dispositivos de salud mental. También refleja cómo se relacionan claramente dependencias a determinadas sustancias con trastornos mentales concretos, lo que es relevante cara a la exploración diagnóstica y al diseño del tratamiento adecuado y personalizado.

En definitiva, se aporta información actualizada sobre la coexistencia real de trastornos adictivos y otros trastornos mentales en el sistema sanitario español, reflejando esta comorbilidad en condiciones de práctica clínica habitual. No obstante, es una primera aproximación a la situación epidemiológico-asistencial del problema, y es evidente que se necesitan más investigaciones para profundizar en su conocimiento y así poder adaptar la respuesta asistencial a la realidad, y con mayor eficiencia.

\section{Reconocimientos}

Al Plan Nacional sobre Drogas, por el apoyo económico y científico: Este estudio ha sido financiado por el PNSD en la convocatoria de subvención para el desarrollo de Programas Supracomunitarios del año 2019.

A todos los profesionales que han participado en la realización de la encuesta, de casi todas las CC. AA, muchos de ellos socios de Socidrogalcohol, por su tiempo y dedicación especialmente en momentos de intensa actividad asistencial por la pandemia de COVID.

\section{Conflicto de intereses}

Este estudio y sus autores no tienen conexión alguna con la industria del tabaco, alcohol o industria farmacéutica, no teniendo ningún conflicto de intereses para su realización.

\section{Referencias}

Araos, P., Vergara-Moragues, E., González-Sáiz, F., Pedraz, M., García-Marchena, N., Romero-Sanchiz, P.,... Rodríguez De Fonseca, F. (2017). Differences in the rates of drug polyconsumption and psychiatric comorbidity among patients with cocaine use disorders according to 
the mental health service. Journal of Psychoactive Drugs, 49, 306-315. doi:10.1080/02791072.2017.1342151.

Arias, F., Ochoa, E. y López-Ibor, J.J. (1996). Trastornos de personalidad en dependientes de opiáceos en tratamiento con naltrexona. Psiquiatría Biológica, 3, 45-52.

Arias, F., Szerman, N., Vega, P., Mesias, B., Basurte, I., Morant, C.,... Babín, F. (2013a). Estudio Madrid sobre prevalencia y características de los pacientes con patología dual en tratamiento en las redes de salud mental y de atención al drogodependiente. Adicciones, 25, 118- 127. doi:10.20882/adicciones.59.

Arias, F., Szerman, N., Vega, P., Mesias, B., Basurte, I., Morant, C.,... Babin, F. (2013b). Alcohol abuse or dependence and other psychiatric disorders. Madrid study on the prevalence of dual pathology. Mental Health and Substance Use, 6, 339-350. doi:10.1080/17523281.2012. 748674.

Arias, F., Szerman, N., Vega, P., Mesias, B., Basurte, I., Morant, C.,... Babin, F. (2013c). Abuso o dependencia a la cocaína y otros trastornos psiquiátricos. Estudio Madrid sobre la prevalencia de la patología dual. Revista de Psiquiatría y Salud Mental, 6, 121-128. doi:10.1016/j. rpsm.2012.09.002.

Arias, F., Szerman, N., Vega, P., Mesías, B., Basurte, I. y Rentero, D. (2016). Trastorno bipolar y trastorno por uso de sustancias. Estudio Madrid sobre prevalencia de patología dual. Adicciones, 29, 186-194. doi:10.20882/ adicciones. 782 .

Becoña Iglesias, E., García García, V., De Echave Sanz, J., Fernández Miranda, J. J., Sánchez Fernández, M. M., Terán Prieto, A.,... Castillo, A. (2006). Tratamiento del tabaquismo en los centros de drogodependencias y alcoholismo en España. Estado de la situación y perspectivas futuras. Adicciones, 18, 23-38. doi:10.20882/adicciones.353.

Compton, W. M., Thomas, Y. F., Stinson, F. S. y Grant, B. F. (2007). Prevalence, correlates, disability, and comorbidity of DSM-IV drug abuse and dependence in the United States. Archives of General Psychiatry, 64, 566-576. doi:10.1001/archpsyc.64.5.566.

Conway, K. P., Compton, W., Stinson, F. S. y Grant, B. F. (2006). Lifetime comorbidity of DSM-IV mood and anxiety disorders and specific drug use disorders. The Journal of Clinical Psychiatry, 67, 247-258. doi:10.4088/ JCP.v67n0211.

Farren, C. K., Hill, K. P. y Weiss, R. D. (2012). Bipolar disorder and alcohol use disorder: A review. Current Psychiatry Reports, 14, 659-666. doi:10.1007/s11920-012-0320-9.

Fatséas, M., Denis, C., Lavie, E. y Auriacombe, M. (2010). Relationship between anxiety disorders and opiate dependence. A systematic review of the literature. Journal of Substance Abuse Treatment, 38, 220-230. doi:10.1016/j. jsat.2009.12.003.
Fernández-Miranda, J. J. (2002). Trastornos de personalidad y adicción: Relaciones etiológicas y conscuencias terapeúticas. Anales de Psiquiatría, 18, 421-427.

Fernández-Miranda, J. J., García-Portilla González, M. P., Sáiz Martínez, P. A., Gutiérrez-Cienfuegos, E. y Bobes García, J. (2001). Influencia de los trastornos psiquiátricos en la efectividad de un programa de mantenimiento prolongado con metadona. Actas Españolas de Psiquiatría, 29, 228-232.

Fernández-Miranda, J. J. y Gutiérrez-Cienfuegos, E. (2005). Trastornos de personalidad y dependencia de heroína: Una relación por determinar. Adicciones, 17, 179-188.

Fernández-Miranda, J. J., Orengo-Caus, T. y Díaz-Fernández, S. (2019). Comorbilidad psiquiátrica en adicciones. Trastorno por uso de opioides y otro trastorno mental comórbido. Guías clínicas basadas en la evidencia. Trastornos por uso de sustancias y otros trastornos mentales. Socidrogalcohol. Valencia, 2019.

Flórez-Menéndez, G., Balcells-Oliveró, M. y Uzal-Fernández, C. (2018). Comorbilidad psiquiátrica en adicciones. Trastorno por uso de alcohol y otro trastorno mental comórbido. Guías clínicas basadas en la evidencia. Trastornos por uso de sustancias y otros trastornos mentales. Socidrogalcohol. Valencia, 2017.

Grant, B. F., Stinson, F. S., Dawson, D. A., Chou, S. P., Dufour, M. C., Compton, W.,... Kaplan, K. (2004). Prevalence and co-occurrence of substance use disorders and independentmood and anxiety disorders. Archives of General Psychiatry, 61, 807-816. doi:10.1001/archpsyc.61.8.807.

Gual, A. (2007). Dual diagnosis in Spain. Drug and Alcohol Review, 26, 65-71.

Guardia-Serecigni, J. y Flórez-Menéndez, G. (2018). Comorbilidad psiquiátrica en adicciones. Trastorno por uso de ansiolíticos e hipnóticos y otro trastorno mental comórbido. Guías clínicas basadas en la evidencia. Trastornos por uso de sustancias y otros trastornos mentales. Socidrogalcohol. Valencia, 2018.

Haro, G., Bolinches, F. y De Vicente, P. (2001). Influencia de los trastornos de la personalidad en la evolución de la conducta adictiva y psicopatología asociada. Adicciones, 13, 93-94.

Hasin, D. S. y Grant, B. F. (2015). The National Epidemiologic Survey on Alcohol and Related Conditions (NESARC) waves 1 and 2: Review and summary of findings. Social Psychiatry and Psychiatric Epidemiology, 50, 16091640. doi:10.1007/s00127-015-1088-0.

Herrero, M. J., Domingo-Salvany, A., Torrens, M. y Brugal, M. T. (2008). Psychiatric comorbidity in young cocaine users: Induced versus independent disorders. Addiction, 103, 284-293. doi:10.1111/j.1360-0443.2007.02076.x.

Hunt, G. E., Large, M. M., Cleary, M., Lai, H. M. X. y Saunders, J. B. (2018). Prevalence of comorbid substance use in schizophrenia spectrum disorders in community 
and clinical settings, 1990-2017: Systematic review and meta-analysis. Drug and Alcohol Dependence, 191, 234-258. doi:10.1016/j.drugalcdep.2018.07.011.

Kessler, R. C. y Merikangas, K. R. (2004). The National Comorbidity Survey Replication (NCS-R): Background and aims. International Journal of Methods in Psychiatric Research, 13, 60-68. doi:10.1002/mpr.166.

Lana, F., Fernández San Martín, M. I., Sánchez Gil, C. y Bonet, E. (2008). Study of personality disorders and the use of services in the clinical population attended in the mental health network of a community area. Actas Españolas de Psiquiatría, 36, 331-336.

Lev-Ran, S., Imtiaz, S., Rehm, J. y Le Foll, B. (2013). Exploring the association between lifetime prevalence of mental illness and transition from substance use to substance use disorders: Results from the National Epidemiologic Survey of Alcohol and Related Conditions (NESARC). The American Journal on Addictions, 22, 93-98.

Martínez-Gras, I., Ferre Navarrete, F., Pascual Arriazu, J., Peñas Pascual, J., De Iceta Ruiz de Gauna, M., Fraguas Herráez, D.,... GIPA, O. (2016). Psychiatric comorbidity in a sample of cocaine-dependent outpatients seen in the Community of Madrid drug addiction care network. Adicciones, 28, 6-12. doi:10.20882/adicciones.785.

Nocon, A., Bergé, D., Astals, M., Martín-Santos, R. y Torrens, M. (2007). Dual diagnosis in an inpatient drug-abuse detoxification unit. European Addiction Research, 13, 192-200. doi:10.1159/000104881.

Ochoa-Mangado, E., Molins-Pascual, S. y Seijo-Ceballos, P. (2018). Comorbilidad psiquiátrica en adicciones. Trastorno por uso de cocaína y otro trastorno mental comórbido. Guías clínicas basadas en la evidencia. Trastornos por uso de sustancias y otros trastornos mentales. Socidrogalcohol. Valencia, 2018.

Pascual-Pastor, F., Fernández-Miranda, J. J., Díaz-Fernández, S. y Sala-Añó, C. (2017). Comorbilidad psiquiátrica en adicciones. Concepto, epidemiología y diagnóstico. Marco general de tratamiento. Guías clínicas basadas en la evidencia. Trastornos por uso de sustancias y otros trastornos mentales. Socidrogalcohol. Valencia, 2017.

Pedrero-Pérez, E. (2018). Trastornos de la personalidad en personas con adicción: Conglomerados diagnósticos y variables psicológicas asociadas. Behavioral Psychology/ Psicologia Conductual, 26, 473-493.

Pereiro, C., Pino, C., Flórez, G., Arrojo, M. y Becoña, E. (2013). Psychiatric comorbidity in patients from the addictive disorders assistance units of Galicia: The COPSIAD study. PLoS ONE, 8. doi:10.1371/journal. pone.0066451.

Regier, D. A., Farmer, M. E., Rae, D. S., Locke, B. Z., Keith, S. J., Judd, L. L. y Goodwin, F. K. (1990). Comorbidity of mental disorders with alcohol and other drug abuse. Results from the Epidemiologic Catchment Area (ECA) Study. JAMA, 264, 2511-2518.
Rodríguez-Jiménez, R., Aragüés, M., Jiménez-Arriero, M.Á., Ponce, G., Muñoz, A., Bagney, A.,... Palomo, T. (2008). Patología dual en pacientes psiquiátricos hospitalizados: Prevalencia y características generales. Invest. clin, 49, 195-205.

Rodríguez-Llera, M. C., Domingo-Salvany, A., Brugal, M. T., Silva, T. C., Sánchez-Niubó, A. y Torrens, M. (2006). Psychiatric comorbidity in young heroin users. Drug and Alcohol Dependence, 84, 48-55. doi:10.1016/j.drugalcdep.2005.11.025.

Roncero, C., Fuste, G., Barral, C., Rodríguez-Cintas, L., Martínez-Luna, N., Eiroa-Orosa, F. y Casas, M. (2011). Therapeutic management and comorbidities in opiate-dependent patients undergoing a replacement therapy programme in Spain: The PROTEUS study. Heroin Addiction and Related Clinical Problems, 13, 5-16.

Sáiz Martínez, P. A., Jiménez Treviño, L., Díaz Mesa, E. M., García-Portilla González, M. P., Marina González, P., Al-Halabí, S.,... Ruiz, P. (2014). Patología dual en trastornos de ansiedad: Recomendaciones en el tratamiento farmacológico. Adicciones, 26, 254-274. doi:10.20882/ adicciones.7.

Sánchez-Peña, J. F., Álvarez-Cotoli, P. y Rodríguez-Solano, J. J. (2012). Psychiatric disorders associated with alcoholism: 2 year follow-up of treatment. Actas Españolas de Psiquiatría, 40, 129-135.

Smith, J. P. y Randall, C. L. (2012). Anxiety and alcohol use disorders: Comorbidity and treatment considerations. Alcohol Research : Current Reviews, 34, 414-431.

Torrens, M., Mestre-Pintó, J. y Domingo-Salvany, A. (2015). Comorbidity of substance use and mental disorders in Europe. Lisbon: EMCDDA. Recuperado de https://www.emcdda.europa.eu/system/files/publications/1988/TDXD15019ENN.pdf.

Welsh, J. W., Knight, J. R., Hou, S. S.-Y., Malowney, M., Schram, P., Sherritt, L. y Boyd, J. W. (2017). Association between substance use diagnoses and psychiatric disorders in an adolescent and young adult clinic-based population. Journal of Adolescent Health, 60, 648-652. doi:10.1016/j.jadohealth.2016.12.018. 